\title{
ON OPTIMAL TEMPERATURE PATHS FOR THERMORHEOLOGICALLY SIMPLE VISCOELASTIC MATERIALS*
}

\author{
BY \\ MORTON E. GURTIN AND LEA F. MURPHY \\ Carnegie-Mellon University
}

Introduction. When a fiber-reinforced viscoelastic material is cooled from cure temperature to room temperature the different coefficients of thermal expansion of the matrix and fibers lead to residual stresses. To study this phenomenon in detail, we consider a thin, isotropic, thermorheologically simple, linearly viscoelastic plate reinforced by a random system of fibers lying in the plane of the plate. We ask the following question: of all temperature paths $\theta(t), 0 \leq t \leq T$, which take on prescribed values at $t=0$ and $t=T$, is there a path which renders the residual stress at $t=T$ a minimum ? ${ }^{1}$

The Euler equation associated with the above problem is a nonlinear integral equation, which we use to show that smooth minimizers are generally not possible (cf. [1]). Indeed, solutions of the Euler equation exhibit a jump discontinuity at $t=0$, are smooth during $(0, T)$, and generally suffer a second jump discontinuity at $t=T^{2}$

For a Maxwell material with an exponential shift function we are able to solve the Euler equation in closed form. We use this solution to compute the optimal temperature path for polymethyl methacrylate with initial and final temperatures $90^{\circ} \mathrm{C}$ and $80^{\circ} \mathrm{C}$, respectively, and with $T=5$ hours. The optimal path produces a residual stress of $32 \mathrm{psi}$ as compared to $220 \mathrm{psi}$ for a linear path.

1. Basic equations. Under the assumption of thermorheologically simple behavior, the stress-strain relation for a homogeneous, isotropic linearly viscoelastic material is ${ }^{3}$

$$
\begin{aligned}
\sigma_{i j}(x, t)= & 2 \int_{-\infty}^{t} \mu\left(\xi_{t}-\xi_{s}\right) \dot{\varepsilon}_{i j}(x, s) d s \\
& +\delta_{i j} \int_{-\infty}^{t} \lambda\left(\xi_{t}-\xi_{s}\right) \dot{\varepsilon}_{k k}(x, s) d s \\
& -\delta_{i j} \int_{-\infty}^{t}\left[2 \mu\left(\xi_{t}-\xi_{s}\right)+3 \lambda\left(\xi_{t}-\xi_{s}\right)\right] \alpha_{m} \dot{\theta}(x, s) d s,
\end{aligned}
$$

* Received October 1, 1979.

${ }^{1}$ This problem was first studied by Weitsman and Ford [1], who developed a numerical procedure for its solution.

${ }^{2}$ Our results are similar to those of Breuer [2], Gurtin, MacCamy and Murphy [3], and Gurtin and Murphy [4], who determine (within the framework of isothermal linear viscoelasticity) strain paths which require least work; the resulting optimal paths suffer jump discontinuities at the endpoints of the prescribed time intervals.

${ }^{3}$ See, for example, Muki and Sternberg [5], Eq. (40). We use Cartesian coordinates and standard indicial notation. A superposed dot indicates differentiation with respect to time. 
where $\sigma_{i j}$ are the components of the stress tensor, $\varepsilon_{i j}$ are the components of the strain tensor, $\theta$ is the temperature, $\mu$ is the shear relaxation function, $2 \mu+3 \lambda$ is the bulk relaxation function, and $\alpha_{m}$ is the (constant) coefficient of thermal expansion. Further,

$$
\xi_{t}=\xi(x, t)=\int_{0}^{t} \phi(\theta(x, r)) d r, \quad \xi_{s}=\xi(x, s),
$$

with $\phi$ the shift factor. Note that

$$
\xi_{t}-\xi_{s}=\int_{s}^{t} \phi(\theta(x, r)) d r
$$

Consider now an infinite viscoelastic plate (here we follow the formulation of Weitsman and Ford [1]) which occupies the region

$$
\left\{x \mid x_{1}, x_{2} \in(-\infty, \infty), x_{3} \in[-h, h]\right\}
$$

and which is reinforced by a system of fibers distributed randomly in planes $x_{3}=$ constant. We assume that the temperature of the plate is independent of position, the surface traction vanishes on the faces $x_{3}= \pm h$, and the plate is unstrained for all negative time. We further assume that the fibers are perfectly bonded to the viscoelastic matrix, so that the strain in the fibers is identical to the strain in the matrix. Finally, we suppose that

$$
\varepsilon_{11}(t)=\varepsilon_{22}(t)=\alpha_{f}[\theta(t)-\theta(0)]
$$

where $\alpha_{f}$ is the (constant) coefficient of thermal expansion of the fibers. (Assumption (3) is reasonable when the fibers are sufficiently stiff relative to the matrix.)

We restrict our attention to solutions with $\sigma_{11}$ and $\sigma_{22}$ the only non-zero components of stress. Then (1) and (3) yield

$$
\begin{aligned}
\sigma(t)=\sigma_{11}(t)=\sigma_{22}(t)= & 2 \int_{0}^{t} \mu\left(\xi_{t}-\xi_{s}\right) \alpha_{f} \dot{\theta}(s) d s \\
& +\int_{0}^{t} \lambda\left(\xi_{t}-\xi_{s}\right)\left[2 \alpha_{f} \dot{\theta}(s)+\dot{\varepsilon}_{33}(s)\right] d s \\
& -\int_{0}^{t}\left[2 \mu\left(\xi_{t}-\xi_{s}\right)+3 \lambda\left(\xi_{t}-\xi_{s}\right)\right] \alpha_{m} \dot{\theta}(s) d s, \\
0=2 \int_{0}^{t} \mu\left(\xi_{t}-\xi_{s}\right) \dot{\varepsilon}_{33}(s) d s+\int_{0}^{t} \lambda\left(\xi_{t}-\xi_{s}\right)\left[2 \alpha_{f} \dot{\theta}(s)+\dot{\varepsilon}_{33}(s)\right] d s & -\int_{0}^{t}\left[2 \mu\left(\xi_{t}-\xi_{s}\right)+3 \lambda\left(\xi_{t}-\xi_{s}\right)\right] \alpha_{m} \dot{\theta}(s) d s .
\end{aligned}
$$

Changing the variable of integration in $(4)_{2}$ from $s$ to $\xi=\xi_{s}$ transforms the equation into a sum of convolutions, and application of the Laplace transform yields

$$
\int_{0}^{t} \lambda\left(\xi_{t}-\xi_{s}\right) \dot{\varepsilon}_{33}(s) d s=\int_{0}^{t} \eta\left(\xi_{t}-\xi_{s}\right) \dot{\theta}(s) d s
$$


where ( $\mathscr{L}$ denotes the Laplace transform; $\mathscr{L}^{-1}$ is the inverse transform)

$$
\left.\eta=\mathscr{L}^{-1} \mid \frac{\mathscr{L}(\lambda)\left[\left(3 \alpha_{m}-2 \alpha_{f}\right) \mathscr{L}(\lambda)+2 \alpha_{m} \mathscr{L}(\mu)\right]}{2 \mathscr{L}(\mu)+\mathscr{L}(\lambda)}\right\} .
$$

Thus $(4)_{1}$ takes the form

$$
\sigma(t)=-\int_{0}^{t} G\left(\xi_{t}-\xi_{s}\right) \dot{\theta}(s) d s,
$$

where

$$
G=2\left(\alpha_{m}-\alpha_{f}\right) \mu+\left(3 \alpha_{m}-2 \alpha_{f}\right) \lambda-\eta .
$$

Note that for the special case in which Poisson's ratio $v=\lambda / 2(\mu+\lambda)$ is constant, ${ }^{4}$

$$
G=2\left(\frac{1+v}{1-v}\right)\left(\alpha_{m}-\alpha_{f}\right) \mu .
$$

(Actually, under the assumption that $v$ is constant, (5) and (7) can be derived from (4) without the use of Laplace transforms.)

2. Optimal temperature paths. Suppose that the plate must be cooled from a given initial temperature $\theta_{0}$ to a final temperature $\theta_{T}$ in a specified time interval $[0, T]$. Our objective is to minimize the residual stress in the plate at the end of the cooling period. In view of (5), the stress at time $T$ is given by

$$
\sigma_{T}(\theta)=-\int_{0}^{T} G\left(\psi_{t}(\theta)\right) \dot{\theta}(t) d t,
$$

where $G$ is defined by (6) and

$$
\psi_{t}(\theta)=\int_{t}^{T} \phi(\theta(s)) d s
$$

It is tacit in (8) that $\theta$ be differentiable; thus our search for a temperature path which minimizes $\left|\sigma_{T}\right|$ begins within the class

$$
\mathscr{A}=\left\{\theta \in C^{1}[0, T] \mid \theta(0)=\theta_{0} \quad \text { and } \quad \theta(T)=\theta_{T}\right\} ;
$$

that is, within the class of smooth temperature paths. Our first result shows that there are no nontrivial optimal paths within this class. We call a path $\theta$ a trivial minimizer if $\sigma_{T}(\theta)=0$. We assume that

$$
G \in C^{1}\left(\mathbb{R}^{+}\right) \text {with } G^{\prime}<0, \quad \phi \in C^{1}(\mathbb{R}) \text { with } \phi>0 .
$$

THEOREM. There are no nontrivial minimizers for $\left|\sigma_{T}\right|$ within $\mathscr{A}$.

Proof. Suppose $\theta \in \mathscr{A}$ minimizes $\left|\sigma_{T}\right|$ and $\sigma_{T}(\theta) \neq 0$. Then for all $\beta \in \mathscr{A}$,

$$
\sigma_{T}(\theta)^{2} \leq \sigma_{T}(\beta)^{2},
$$

\footnotetext{
${ }^{4}$ Typically, $\alpha_{m}>\alpha_{f}$, so that for $v$ constant $G$ is a positive multiple of $\mu$.
} 
and the variation

$$
\delta \sigma_{T}(\theta)^{2}[\omega]=\left.\frac{d}{d \zeta} \sigma_{T}(\theta+\zeta \omega)^{2}\right|_{\zeta=0}
$$

vanishes for every $\omega \in C^{1}[0, T]$ satisfying $\omega(0)=0=\omega(T)$. But

$$
\delta \sigma_{T}(\theta)^{2}[\omega]=2 \sigma_{T}(\theta) \delta \sigma_{T}(\theta)[\omega] ;
$$

thus

$$
\delta \sigma_{T}(\theta)[\omega]=0
$$

for all smooth functions $\omega$ which vanish at 0 and $T$. Let $\omega$ be one such function. Then

$$
\delta \sigma_{T}(\theta)[\omega]=-\int_{0}^{T} G\left(\psi_{t}\right) \dot{\omega}(t) d t-\int_{0}^{T} G^{\prime}\left(\psi_{t}\right) \dot{\theta}(t) \int_{t}^{T} \phi^{\prime}(\theta(s)) \omega(s) d s d t,
$$

where we have written $\psi_{t}$ for $\psi_{t}(\theta)$. The first term is easily integrated by parts to give

$$
-\int_{0}^{T} \omega(t) G^{\prime}\left(\psi_{t}\right) \phi(\theta(t)) d t
$$

and letting

$$
F(t)=\int_{0}^{t} G^{\prime}\left(\psi_{s}\right) \dot{\theta}(s) d s
$$

the second term yields

$$
-\int_{0}^{T} \dot{F}(t) \int_{t}^{T} \phi^{\prime}(\theta(s)) \omega(s) d s d t=-\int_{0}^{T} F(t) \phi^{\prime}(\theta(t)) \omega(t) d t .
$$

Thus

$$
\left.\delta \sigma_{T}(\theta)[\omega]=-\int_{0}^{T} \mid G^{\prime}\left(\psi_{t}\right) \phi(\theta(t))+\phi^{\prime}(\theta(t)) \int_{0}^{t} G^{\prime}\left(\psi_{s}\right) \dot{\theta}(s) d s\right\} \omega(t) d t,
$$

and this expression can equal zero for all suitable $\omega$ only if

$$
G^{\prime}\left(\psi_{t}\right) \phi(\theta(t))+\phi^{\prime}(\theta(t)) \int_{0}^{t} G^{\prime}\left(\psi_{s}\right) \dot{\theta}(s) d s=0
$$

for almost every $t \in[0, T]$. But the left side of this equation is a continuous function of $t$ which is not zero at $t=0$. Thus we have a contradiction and the proof is complete.

It is clear from the preceding theorem that if $\left|\sigma_{T}\right|$ is to be minimized, either a temperature path producing zero stress must be found, or else the class of functions for which $\sigma_{T}$ is defined must be enlarged. For many problems there is no temperature path producing zero stress. Consequently we are motivated to extend ${ }^{5}$ the functional $\sigma_{T}$.

\footnotetext{
${ }^{5}$ Our procedure here parallels that used in the treatment of the optimization problems considered in [3] and [4].
} 
If we integrate (8) by parts, using the boundary conditions $\theta(0)=\theta_{0}$ and $\theta(T)=\theta_{T}$, we arrive at

$$
\hat{\sigma}_{T}(\theta)=-\int_{0}^{T} G^{\prime}\left(\psi_{t}(\theta)\right) \phi(\theta(t)) \theta(t) d t+\theta_{0} G\left(\psi_{0}\right)-\theta_{T} G(0) .
$$

If $\theta \in \mathscr{A}, \hat{\sigma}_{T}(\theta)=\sigma_{T}(\theta)$. But $\hat{\sigma}_{T}$ is also defined for any function $\theta$ in

$$
\mathscr{C}=\text { the class of bounded measurable functions on }[0, T] \text {. }
$$

Recall that when Poisson's ratio is constant, $G$ is, by (7), essentially the shear relaxation function. We therefore take, as prototypes for $G$ and $\phi$,

$$
\begin{aligned}
G(t) & =G_{\infty}+\left(G_{0}-G_{\infty}\right) e^{-t / \tau} \\
\phi(\xi) & =\phi_{0} e^{\rho \xi}
\end{aligned}
$$

with $G_{0}, G_{\infty}, \tau, \phi_{0}$ and $\rho$ positive constants. We shall discuss the above problem for general functions $\mu$ and $\phi$ under the following hypotheses, which are satisfied by the functions (9):

$$
\begin{aligned}
& \left(\mathrm{H}_{1}\right) \quad G \in C^{2}\left(\mathbb{R}^{+}\right), G^{\prime}<0, G^{\prime \prime}>0, \\
& \left(\mathrm{H}_{2}\right) \quad \phi \in C^{3}(\mathbb{R}), \phi>0, \phi^{\prime}>0, \\
& \left(\mathrm{H}_{3}\right) \phi^{2} / \phi^{\prime} \text { is a strictly increasing function. }
\end{aligned}
$$

As in the proof of the first theorem, $\theta \in \mathscr{C}$ minimizes $\left|\hat{\sigma}_{T}\right|$ only if

$$
\hat{\sigma}_{t}(\theta) \delta \hat{\sigma}_{T}(\theta)[\omega]=0
$$

for all $\omega \in \mathscr{C}$. If there is a trivial minimizer $\theta \in \mathscr{C}$ (i.e., a $\theta$ with $\hat{\sigma}_{T}(\theta)=0$ ), then $\theta$ is the optimal path. However, for many real problems, no such $\theta$ exists. To see this assume for the moment that

$$
k=\inf _{\xi \in \mathbb{B}} \xi \phi(\xi)
$$

exists. ${ }^{6}$ Then $\left(\mathrm{H}_{1}\right)$ and $\left(\mathrm{H}_{2}\right)$ imply

$$
\hat{\sigma}_{T}(\theta) \geq \theta_{0} G(\infty)-\theta_{T} G(0)-T k G^{\prime}(0)
$$

for all $\theta$ in $\mathscr{C}$. Thus if $G>0$ and the difference between the initial and final temperatures is large, $\hat{\sigma}$ is bounded below by a positive number and no $\theta \in \mathscr{C}$ can produce zero residual stress.

In the case where $\hat{\sigma}_{T}$ is bounded away from zero, $\left|\hat{\sigma}_{T}\right|$ can be minimized only by extreme points of $\hat{\sigma}_{T}$. The next theorem, which is based on hypotheses $\left(\mathrm{H}_{1}\right)-\left(\mathrm{H}_{3}\right)$, characterizes such extreme points.

THEOREM. Let $\theta \in \mathscr{C}$ be a nontrivial minimizer of $\left|\hat{\sigma}_{T}\right|$. Then $\theta$ is essentially ${ }^{7}$ a continuous, strictly decreasing function with ${ }^{8}$

$$
\theta(0)<\theta_{0}
$$

\footnotetext{
${ }^{6}$ Indeed, if $\phi$ is given by $(9)_{2}$, then $k=-\phi_{0} / \rho e$.

${ }^{7}$ I.e., up to a set of measure zero.

${ }^{8}$ Here, $\theta(0)=$ ess $\lim _{t \downarrow 0} \theta(t)$.
} 
Proof. Suppose $\theta \in \mathscr{C}$ minimizes $\left|\hat{\sigma}_{T}\right|$ and $\hat{\sigma}_{T}(\theta) \neq 0$. Then

$$
\delta \hat{\sigma}_{T}(\theta)[\omega]=0
$$

for all $\omega \in \mathscr{C}$. For any such $\omega$

$$
\begin{aligned}
\delta \hat{\sigma}_{T}(\theta)[\omega]= & -\int_{0}^{T} G^{\prime \prime}\left(\psi_{t}\right) \phi(\theta(t)) \theta(t) \cdot \int_{t}^{T} \phi^{\prime}(\theta(s)) \omega(s) d s d t \\
& -\int_{0}^{T} G^{\prime}\left(\psi_{t}\right)\left[\phi(\theta(t))+\phi^{\prime}(\theta(t)) \theta(t)\right] \omega(t) d t \\
& +\theta_{0} G^{\prime}\left(\psi_{0}\right) \int_{0}^{T} \phi^{\prime}(\theta(t)) \omega(t) d t,
\end{aligned}
$$

and letting

$$
F(t)=\int_{0}^{t} G^{\prime \prime}\left(\psi_{s}\right) \phi(\theta(s)) \theta(s) d s
$$

the first term becomes

$$
-\int_{0}^{T} \dot{F}(t) \int_{t}^{t} \phi^{\prime}(\theta(s)) \omega(s) d s d t=-\int_{0}^{T} F(t) \phi^{\prime}(\theta(t)) \omega(t) d t
$$

hence

$$
\begin{aligned}
\delta \hat{\sigma}_{T}(\theta)[\omega]= & -\left\{\int_{0}^{T}\left[\int_{0}^{T} G^{\prime \prime}\left(\psi_{s}\right) \phi(\theta(s)) \theta(s) d s-\theta_{0} G^{\prime}\left(\psi_{0}\right)\right)\right. \\
& \left.+G^{\prime}\left(\psi_{t}\right)\left(\theta(t)+\frac{\phi(\theta(t))}{\phi^{\prime}(\theta(t))}\right)\right] \phi^{\prime}(\theta(t)) \omega(t) d t !
\end{aligned}
$$

Hence $\theta$ must satisfy the Euler equation

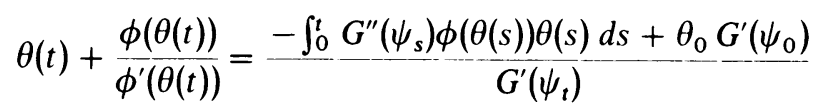

for almost all $t$ in $[0, T] .\left(\mathrm{H}_{1}\right)$ and $\left(\mathrm{H}_{2}\right)$ imply that the right side of (11) is essentially continuously differentiable. ${ }^{9}\left(\mathrm{H}_{3}\right)$ implies that

$$
\xi+\left(\phi(\xi) / \phi^{\prime}(\xi)\right)
$$

is a strictly increasing function of $\xi$. Thus $\theta$ is essentially continuously differentiable on $[0, T]$, and we may differentiate (11) with respect to $t$; the result is

$$
\dot{\theta}(t)=\frac{G^{\prime \prime}\left(\psi_{t}\right) \phi(\theta(t))^{2} \phi^{\prime}(\theta(t))}{G^{\prime}\left(\psi_{t}\right)\left[2 \phi^{\prime}(\theta(t))^{2}-\phi(\theta(t)) \phi^{\prime \prime}(\theta(t))\right]} .
$$

Thus $\left(\mathrm{H}_{1}\right)-\left(\mathrm{H}_{3}\right)$ imply that

$$
\dot{\theta}(t)<0
$$

\footnotetext{
${ }^{9}$ Here we mean continuous everywhere and almost everywhere differentiable.
} 
for almost every $t$ in $[0, T]$. Finally, evaluation of (11) at $t=0$ yields

$$
\theta(0)+\frac{\phi(\theta(0))}{\phi^{\prime}(\theta(0))}=\theta_{0},
$$

and (10) follows from $\left(\mathrm{H}_{2}\right)$.

Remark. It is interesting to note that the nontrivial extreme points of $\left|\hat{\sigma}_{T}\right|$ are independent of the final temperature $\theta_{T}$, so that, in general,

$$
\theta(T) \neq \theta_{T} .
$$

The existence of trivial minimizers does, however, depend crucially on the value of $\theta_{T}$.

3. Maxwell materials. In this section we consider a Maxwell material with an exponential shift function and constant Poisson's ratio, i.e. we assume that (9) holds. Then we can find an explicit solution of the integral-differential equation (12) subject to the boundary condition (13). ${ }^{11}$ Indeed, substitution of (9) into (12) and (13) yields

$$
\dot{\theta}=-\frac{\phi_{0}}{\tau \rho} e^{\rho \theta}, \quad \theta(0)=\theta_{0}-\frac{1}{\rho} .
$$

Eq. $(14)_{1}$ is equivalent to

$$
\left(e^{-\rho \theta}\right) \cdot=\phi_{0} / \tau
$$

and hence the unique solution to (14) is

$$
\theta^{*}(t)=-\frac{1}{\rho} \ln \left(\frac{\phi_{0} t}{\tau}+e^{1-\rho \theta_{0}}\right) .
$$

Extreme points of $\hat{\sigma}_{T}$ necessarily solve (14). Thus, unless there is a path producing zero stress, $\theta^{*}$ is the only candidate in $\mathscr{C}$ for a minimizer of $\left|\hat{\sigma}_{T}\right|$. Numerical experiments indicate that $\theta^{*}$ does, in fact, minimize $\left|\hat{\sigma}_{T}\right|$.

At any time $t \in[0, T)$, the stress due to $\theta^{*}$ is

$$
\hat{\sigma}_{t}\left(\theta^{*}\right)=G_{\infty}\left[\theta_{0}-\theta^{*}(t)\right]+\frac{1}{\rho}\left[G_{0}-G_{\infty}\right],
$$

while at time $T$ the stress jumps to the value

$$
\hat{\sigma}_{T}\left(\theta^{*}\right)=\sigma_{T^{-}}\left(\theta^{*}\right)+G_{0}\left[\theta^{*}\left(T^{-}\right)-\theta_{T}\right] .
$$

Remark. As noted before, the path $\theta^{*}$ is independent of the final temperature $\theta_{T}$. Further, as is clear from (15), $\theta^{*}$ for a Maxwell material is also independent of the duration $T$ of the time interval. Thus given an initial temperature $\theta_{0}$, the function $\theta^{*}(t)$, $0 \leq t<\infty$, defined by (15) represents a canonical optimal path; the optimal path for any

${ }^{10}$ Weitsman and Ford [1]. These authors also note that $\theta$ is independent of $\theta_{T}$ and continuous on $(0, T)$.

${ }^{11}$ Assuming that some $\theta(0)$ solves (13), the existence of a solution to the general boundary value problem (12), (13) is guaranteed if for some constant $m$ and all $\xi<\theta(0)$,

$$
\phi(\xi)^{2} \phi^{\prime}(\xi) /\left(2 \phi^{\prime}(\xi)^{2}-\phi(\xi) \phi^{\prime \prime}(\xi)\right) \leq m .
$$


given interval $[0, T]$ is simply the restriction of this function to $[0, T]$. Similarly, $\hat{\sigma}_{t}\left(\theta^{*}\right)$, $0 \leq t<\infty$ defined by (16) is a canonical stress path. Given a final time $T$ and a final temperature $\theta_{T}$, the stress on $[0, T)$ is the restriction of this path to $[0, T)$, while the stress at time $T$ is given by (17).

4. An example. As an example we consider the polymer polymethyl methacrylate. We assume that Poisson's ratio is constant, so that Young's modulus, E, and the shear modulus are related by ${ }^{12}$

$$
\mu(t)=E(t) / 2(1+v)
$$

The coefficient of thermal expansion of the fibers is typically an order of magnitude smaller than $\alpha_{m}$, and for convenience we neglect it here. Then (7) yields

$$
G(t)=\frac{\alpha_{m}}{1-v} E(t)
$$

Experimental values for $v$ and $\alpha_{m}$ are

$$
v=0.35, \quad \alpha_{m}=8 \times 10^{-5} /{ }^{\circ} \mathrm{C},
$$

while experimental curves for $G$ and $\phi$ are shown in Fig. 1 (the data for polymethyl methacrylate are taken from [5], pp. 203-204).

We approximate the data by functions of the form (9). In particular, we take

$$
G_{0}=38, \quad G_{\infty}=18, \quad \tau=1.2 ;
$$

this yields a function $G$ which approximates the data fairly well in the range $10 \mathrm{~min}$. $\leq$ $t \leq 15$ hrs. (cf. Fig. 1). Similarly,

$$
\phi_{0}=7.5 \times 10^{-4}, \quad \rho=0.09
$$

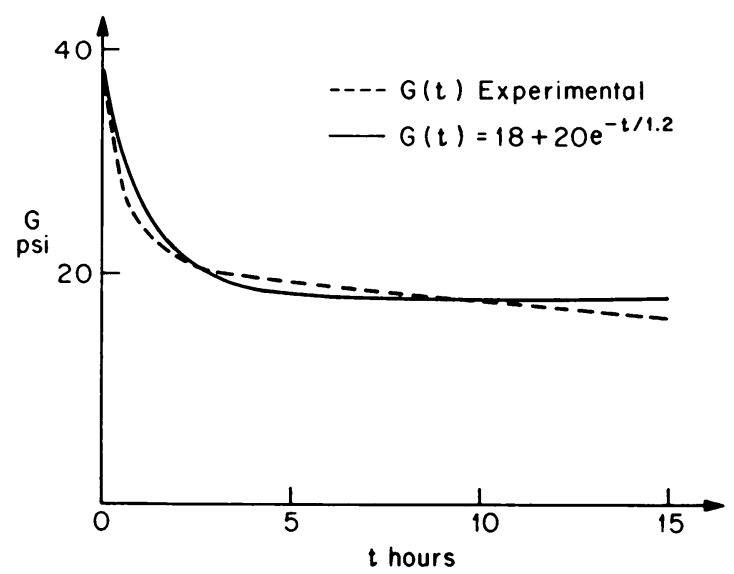

FIG. 1. Experimental and approximate relaxation functions.

12 This relation follows from [5], Eq. (17) $)_{1}$, under the assumption that $v$ is constant. 
yields the approximation shown in Fig. 2, which is reasonable in the range $60^{\circ} \mathrm{C} \leq \theta \leq 95^{\circ} \mathrm{C}$.

The optimal path $\theta^{*}$ corresponding to cooling the plate from $\theta_{0}=90^{\circ} \mathrm{C}$ to $\theta_{T}=80^{\circ} \mathrm{C}$ in a time $T=5$ hours is graphed in Fig. 3. The residual stress corresponding to the optimal path is $32 \mathrm{psi}$, while a linear path produces a stress of $220 \mathrm{psi}$. The stress history corresponding to $\theta^{*}$ is shown in Fig. 4. It is important to note that although the optimal path leads to a lower residual stress than the linear path, the stress due to a linear path increases monotonically, while the stress on the optimal path does not. In fact, in the above example the optimal path is accompanied by a somewhat larger stress (735 psi) during the cooling period. These and other results are summarized in Table 1. (The negative entry in the first row indicates the existence of trivial minimizers for that case.)

5. Other criteria. Thus far we have been concerned solely with the stress in the plate at the final instant of the cooling period. We now consider the behavior of the stress in a

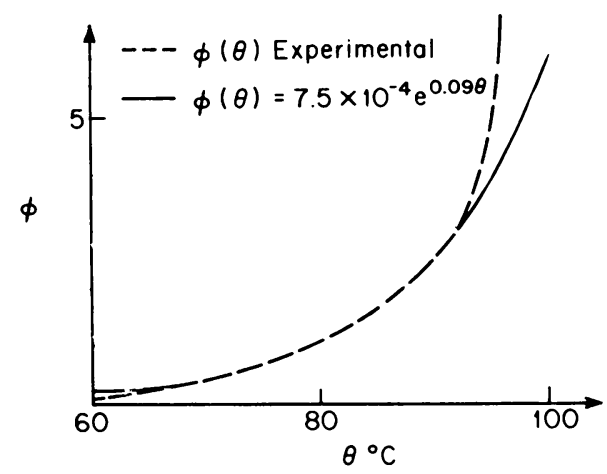

FIG. 2. Experimental and approximate shift factors.

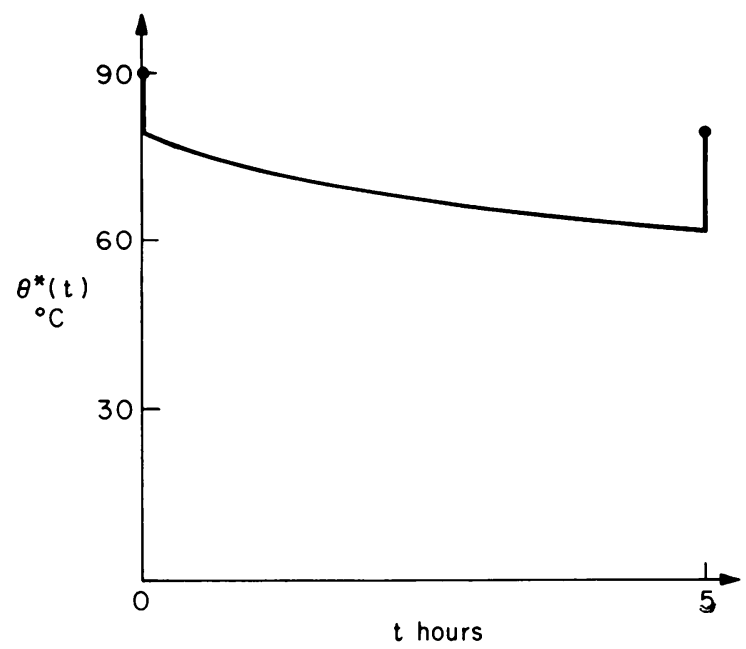

FIG. 3. Optimal temperature path $\theta^{*}$ for $\theta_{0}=90^{\circ} \mathrm{C}, \theta_{T}=80^{\circ} \mathrm{C}$, and $T=5$ hours. 


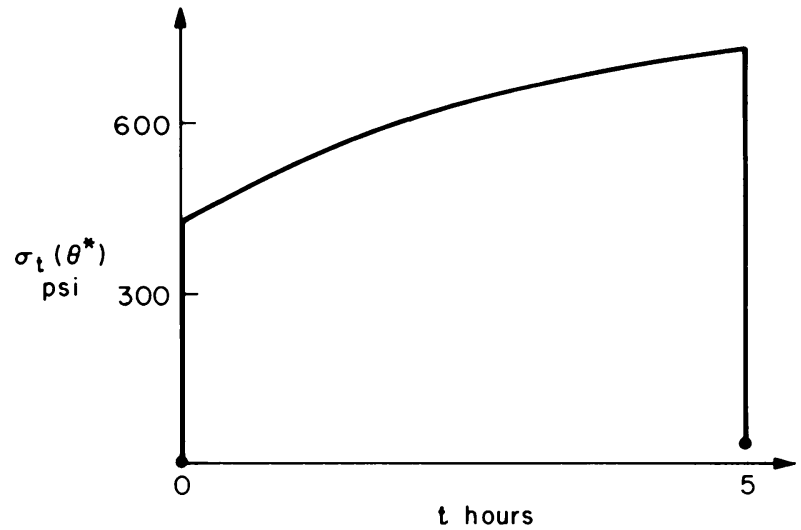

Fig. 4. Stress path corresponding to the optimal path for $\theta_{0}=90^{\circ} \mathrm{C}, \theta_{T}=80^{\circ} \mathrm{C}$, and $T=5$ hours.

TABLE 1.

\begin{tabular}{cccccc}
\hline$\theta_{0}{ }^{\circ} \mathrm{C}$ & $\theta_{T}{ }^{\circ} \mathrm{C}$ & $T$ hrs & $\begin{array}{c}\text { residual stress } \\
\text { optimal path } \\
\text { psi }\end{array}$ & $\begin{array}{c}\text { residual stress } \\
\text { linear path } \\
\text { psi }\end{array}$ & $\begin{array}{c}\text { maximum stress } \\
\text { optimal path } \\
\text { psi }\end{array}$ \\
\hline 90 & 80 & 10 & -98 & 202 & 852 \\
90 & 70 & 10 & 283 & 444 & 852 \\
90 & 80 & 5 & 32 & 220 & 735 \\
90 & 70 & 5 & 412 & 497 & 735 \\
90 & 80 & 1 & 255 & 301 & 535 \\
90 & 70 & 1 & 635 & 654 & 535 \\
\hline
\end{tabular}

time interval $[T, U]$ following the cooling interval. If the temperature of the plate is held constant at $\theta_{T}$ during $[T, U]$, then for any $t \in[T, U]$ and $\theta \in \mathscr{C},(9)$ enables us to write the stress at time $t$ as follows:

$$
\hat{\sigma}_{y}(\theta)=\exp \left\{-(t-T) \phi\left(\theta_{T}\right) / \tau\right\}\left(\hat{\sigma}_{T}(\theta)-A\right)+A,
$$

where $A=G_{\infty}\left(\theta_{0}-\theta_{T}\right)>0$. We will use (18) to discuss other minimization criteria for the stress. In general, we would expect the optimal path to depend on how we choose to "average" the stress over $[T, U]$. We show, however, that two natural measuring procedures give rise to the same optimal path. In fact, if $\hat{\sigma}_{T}(\beta)>0$ for all $\beta \in \mathscr{C}$, then the following are equivalent:

$$
\begin{aligned}
\hat{\sigma}_{T}(\theta) & \leq \hat{\sigma}_{T}(\beta) \quad \text { for all } \beta \in \mathscr{C} ; \\
\max _{T \leq t \leq U}\left|\hat{\sigma}_{t}(\theta)\right| & \leq \max _{T \leq t \leq U}\left|\hat{\sigma}_{t}(\beta)\right| \text { for all } \beta \in \mathscr{C} ; \\
\int_{T}^{U} \hat{\sigma}_{t}(\theta)^{2} d t & \leq \int_{T}^{U} \hat{\sigma}_{t}(\beta)^{2} d t \quad \text { for all } \beta \in \mathscr{C} .
\end{aligned}
$$

To see that (i) $\Leftrightarrow$ (ii), let $\theta \in \mathscr{C}$ and first suppose that $\hat{\sigma}_{T}(\theta) \geq A$. Then

$$
A \leq \hat{\sigma}_{T}(\theta) \leq \hat{\sigma}_{T}(\beta)
$$


for all $\beta \in \mathscr{C}$, which implies that

$$
\max _{T \leq t \leq U}\left|\hat{\sigma}_{t}(\beta)\right|=\hat{\sigma}_{T}(\beta)
$$

for all $\beta \in \mathscr{C}$, and (ii) holds. Conversely, suppose that (ii) is true, but for some $\bar{\beta} \in \mathscr{C}$,

$$
\hat{\sigma}_{T}(\bar{\beta})<\hat{\sigma}_{T}(\theta)
$$

Then a simple computation shows that

$$
\max _{T \leq t \leq U}\left|\hat{\sigma}_{t}(\bar{\beta})\right| \leq \max _{T \leq t \leq U}\left|\hat{\sigma}_{t}(\theta)\right|
$$

which contradicts (ii). The case $\hat{\sigma}_{T}(\theta)<A$ is similar. We now show (i) $\Leftrightarrow$ (iii). For all $\theta \in \mathscr{C},(18)$ implies that

$$
\int_{T}^{U} \hat{\sigma}_{t}(\theta)^{2} d t=a \hat{\sigma}_{T}(\theta)^{2}+b \hat{\sigma}_{T}(\theta)+c
$$

where

$a=2 \tau\left\{1-\exp \left(-2 \phi\left(\theta_{T}\right)(U-T) / \tau\right)\right\} / \phi\left(\theta_{T}\right)$,

$b=A \tau\left\{1-\exp \left(-\phi\left(\theta_{T}\right)(U-T) / \tau\right)\right\}^{2} / \phi\left(\theta_{T}\right)$,

$c=A^{2}(U-T)+A^{2} \tau\left\{-\frac{3}{2}+2 \exp \left(-\phi\left(\theta_{T}\right)(U-T) / \tau\right)-\frac{1}{2} \exp \left(-2 \phi\left(\theta_{T}\right)(U-T) / \tau\right)\right\} / \phi\left(\theta_{T}\right)$.

Since $a$ and $b$ are positive, the right side of (19) is an increasing function of $\hat{\sigma}_{T}(\theta)$ for $\hat{\sigma}_{T}(\theta)>0$. Thus minimizing (19) over $\mathscr{C}$ is equivalent to minimizing $\hat{\sigma}_{T}$ over $\mathscr{C}$.

Remark. One might also ask the question: What is the optimal length of the cooling period? That is, how can we choose $T$ and $\theta(t), 0 \leq t \leq T$ to minimize $\left|\hat{\sigma}_{T}(\theta)\right|$ ? For a Maxwell material, if we assume that $\theta^{*}$ is indeed optimal, we find that as we increase $T$ the minimum residual stress decreases until, for some $T$, it is zero.

\section{REFERENCES}

[1] Y. Weitsman and D. Ford, On the optimization of cool-down temperatures in viscoelastic resins, Proc. Soc. Engineering Science, 1977.

[2] S. Breuer, Minimizing the strain-rate history and the resulting greatest lower bound on work in linear viscoelasticity, Z. Angew. Math. Mech. 49, 209-213 (1969)

[3] M. E. Gurtin, R. C. MacCamy and L. F. Murphy, On optimal strain paths in linear viscoelasticity, Quart. Appl. Math. 37, 151-156 (1979)

[4] M. E. Gurtin and L. F. Murphy, Optimal strain paths in linear viscoelasticity: the effect of the past history, in Proc. IUT AM Symp. Variational Methods in the Mechanics of Solids, Evanston, Illinois, September, 1978

[5] R. Muki and E. Sternberg, On transient thermal stresses in viscoelastic materials with temperature-dependent properties, J. Appl. Mech. ASME 83, 193-207 (1961) 